\title{
Brettanomyces bruxellensis: Overview of the hidden diversity and complexity of an anthropized yeast
}

\author{
Jules Harrouard ${ }^{1}$, Chris Eberlein ${ }^{2}$, Patricia Ballestra ${ }^{1}$, Marguerite Dols-Lafargue ${ }^{1}$, Isabelle \\ Masneuf-Pomarede ${ }^{1}$, Cecile Miot-Sertier ${ }^{1}$, Joseph Schacherer $^{2}$, and Warren Albertin ${ }^{1}$ \\ ${ }^{1}$ Institut des Sciences de la Vigne et du Vin \\ ${ }^{2}$ GMGM
}

October 18, 2021

\begin{abstract}
Human-associated microorganisms are ideal models to study the impact of environmental changes on species evolution and adaptation. The yeast Brettanomyces bruxellensis is a good example of organism facing anthropogenic-driven selective pressures. It is associated with fermentation processes in which it can be considered either as a spoiler (e.g. winemaking, bioethanol production) or as a beneficial microorganism (e.g. production of specific beers, kombucha). Besides its industrial interests, noteworthy parallels and dichotomies with Saccharomyces cerevisiae propelled B. bruxellensis as a valuable complementary yeast model. In this review, we emphasize that the broad genetic and phenotypic diversity of this species is only beginning to be revealed. Population genomic studies have revealed the co-existence of auto- and allotriploidization events with different evolutionary outcomes. The various diploid, autotriploid and allotriploid subpopulations are associated with specific fermented processes, suggesting independent adaptation phenomena to anthropized environments. Phenotypically, B. bruxellensis is renowned for its ability to metabolize a wide variety of carbon and nitrogen sources, which may explain its ability to colonize already fermented environments showing low-nutrient contents. Several traits of interest could be related to adaptation to human activities (e.g. nitrate metabolization in bioethanol production, resistance to sulphite treatments in winemaking). However, phenotypic traits are insufficiently studied in view of the great genomic diversity of the species. Future work will have to take into account strains of varied substrates, geographical origins as well as displaying different ploidy levels. Finally, we discuss the characteristics of B. bruxellensis which may prove to be of wider interest in future research.
\end{abstract}

\section{Hosted file}

Main Document.docx available at https://authorea.com/users/441614/articles/542070brettanomyces-bruxellensis-overview-of-the-hidden-diversity-and-complexity-of-ananthropized-yeast 